\title{
Lista de Revisores / List of reviewers 2013
}

Acuña, Marcelo (Argentina)

Allende, José Luis (Argentina)

Ambali, Manoj P. (India)

Ang, Eng-Tat (Singapur)

Apaydin, Nihal (Turquía)

Armand Ugon, Gustavo (Uruguay)

Arraez-Aybar, Luis A. (España)

Aughsteen, Adib A. (Jordania)

Barut, Cagatay (Turquía)

Beheiry, Eman E. (Egipto)

Bendersky, Mariana (Argentina)

Buyükmumcu, Mustafa (Turquía)

Campero, A. Álvaro (Argentina)

Ceccón, Gabriel A. F. (Argentina)

Cömert, Ayhan (Turquía)

Criado del Río, Luis E. (Argentina)

David, Oscar P. (Argentina)

Decker, Summer J. (U.S.A.)

Didilescu, Andreea (Rumania)

Elizondo-Omaña, Rodrigo E. (México)

Espósito, Evangelina (Argentina)

Fabrizio, Philip A. (U.S.A.)

Hamel, Antoine (Francia)

Hassanali, Jameela (Kenia)

Haviarová, Zora (Eslovaquia)

Jalalian, Faranak (Irán)

Kervancioglu, Piraye (Turquía)

Krishnan, Subramaniam (Malasia)

Mamatha, H. (India)

Mandiola Lagunas, Eduardo (Chile)

Martinez Benia, Fernando (Uruguay)

Menezes, Ritesh (India)

Musa, Ahmed (Rumania)
Nayak, Soubhagya R. (India)

Olave Riffo, Enrique W. (Chile)

Ozener, Baris (Turquía)

Panagouli, Eleni (Grecia)

Piera, Verónica (España)

Pivtchenko, Petr G. (Bielorusia)

Prada Oliveira, Arturo (España)

Prata, Minati (India)

Przystariska, A (Polonia)

Rojas Oviedo, José D. (Colombia)

Rusu, Mugurel C. (Rumania)

Sankar, Devi K. (India)

Santini, Ario (Reino Unido)

Sañudo, José R. (España)

Sforza, Chiarella (Italia)

Sharma, Alok (India)

Sharma, Kalpana (Nepal)

Shirasaki, Hideaki (Japón)

Sirasangandla, Sreenivasaf R. (India)

Solla, Horacio E. (Uruguay)

Spitale, Luis S. (Argentina)

Thwin, San S. (Malasia)

Totlis, Trifon (Grecia)

Vaiman, Michael (Israel)

Van De Water, Thomas R. (U.S.A.)

Varlik, S. K. (Turquía)

Vasovic, Ljiljana (Serbia)

Verma, Poonam (India)

Vishwanath, Srikanteswara (Antigua)

Vlajkovic, Slobodan (Serbia)

Zabala-Gonzalez, Marco A. (México)

Zahoi, Delia E. (Rumania) 